\title{
WAVE PROPAGATION IN LAYERED ELASTIC MEDIA FOR ANTIPLANE DEFORMATION
}

\author{
CHIEN-CHING MA and KUO-CHENG HUANG \\ Department of Mechanical Engineering, National Taiwan University, Taipei, Taiwan 10764, \\ Republic of China
}

(Received 8 July 1993 ; in revised form 18 February 1994)

\begin{abstract}
In this study, a composite laminate with $n$ layers subjected to dynamic antiplane impact loading on the surface is investigated in detail. The transient responses of stresses and displacement on each layer are expressed in a closed form. For the numerical investigation, a two-layered composite laminate is considered. In analyzing this problem, the reflections and refractions of stress waves must be taken into account, which will generate infinite waves on each composite layer. In some classes of dynamic problem, the ability to find a static field may hinge on waiting for the wave fronts to pass and the transient to die away. The characteristic time after which the transient effect can be neglected is investigated in detail.
\end{abstract}

\section{INTRODUCTION}

The propagation of stress waves through an unbounded medium is not a difficult subject. If the boundary is introduced, however, reflected waves will be generated from the free surface, making the problem more complicated. The classical analysis in this area was first proposed by Lamb (1904); he considered the half-space subjected to point and line loads on the surface of a semi-infinite half-space. Since this early analysis of Lamb, a great many contributions have appeared, pertaining to what is commonly referred to as Lamb's problem. de Hoop (1961) and Cagniard (1962) proposed a powerful and elegantly simple method which is known as the Cagniard-de Hoop technique for inverting transforms in a wide range of elastodynamic wave propagation problems. Buried source problems are of considerable interest in seismology and have been studied by many investigators, including Lamb. Nakano (1925) analyzed the buried line dilatation source in a half-space, but he failed in generalizing his harmonic steady-state results to transient solutions. Lapwood (1949) re-studied Nakano's problem, and later Garvin (1956) also solved Nakano's buried line dilatation source problem by using a suitable distortion of the contour suggested by the work of Cagniard. In Garvin's paper, the numerical results are limited to surface displacement due to a dilatation source, and the generated waves in the medium are incident longitudinal waves, reflected longitudinal waves and shear waves. Recently, Tsai and Ma (1991) obtained the complete transient solutions of buried dynamic point forces and displacement jumps for an elastic half-space.

The applications of composite materials have grown very rapidly for the last two decades because of their high strength and light weight. Composite materials have been widely used in aeronautical industries to replace metals in aircraft structures for the purpose of weight saving. The increasing importance of composite materials in engineering applications has generated a wide interest in the mechanical properties of such media. The methods to find the response of composite laminates under various conditions have appeared to be of most significant concern in this field. The capability of composite materials to transmit impact loads is often the critical feature of design. Composite materials are sometimes chosen for use because of their static properties, and as an auxiliary matter they are required to transmit dynamic loads. A good understanding of their static and dynamic behavior under various loading conditions is needed. For static analysis, this has already received widespread attention in the last two decades. However, the study of transient response of composite materials subjected to dynamic loading has been intensively investigated in recent years. Hence the characterization and methodology for studying wave 
propagation in composite materials is of prime interest. Analytical investigation of the composite materials subjected to impact loading has been meagre because the elastodynamic stress analysis involves numerous parameters and is enormously complex.

Wave propagation in layered media should be studied extensively because of its wide technological applications. A key to attacking these problems involves considering the average stress and strain response to waves whose wavelengths are long compared with the characteristic dimension of the layer. Sun et al. (1968) have developed a continuum theory for composite elastic materials which they have named the "effective modulus" theory. The effective modulus theory is based on the assumption that the layered medium behaves on the whole as a homogeneous but transversely isotropic continuum. Harmonic waves in composites with isotropic layers were also studied by Stern et al. (1971), Hegemier and Nayfeh (1973), Hegemier and Bache (1973), Drumheller and Bedford (1973) and NematNasser and Yamada (1981). Transient plane waves propagating in a periodically layered elastic medium were examined by Ting and Mukunoki (1979), Mukunoki and Ting (1980) and Tang and Ting (1985). In studying the wave propagation in a layered medium, various approximate theorics have been proposed to predict the dynamic response. Most of the approximate theories can predict satisfactorily the frequency equation due to a sinusoidal oscillation and some can also predict the late-time asymptotic solution in a semi-infinite layered medium due to a step load applied at the boundary.

Because of the difficulty in analyzing the transient response of infinite number of reflected and refracted waves in a layered composite structure, only very few papers used the transient analysis to study the phenomena of the wave propagation in a layered medium. For transient waves, one could in principle obtain the solution by superimposing harmonic waves of all frequencies and all modes. This approach, however, is not practical and a more direct approach should be employed to study the transient waves. In this study, the transient response of applying a concentrated antiplane dynamic point force at the top surface of an $n$-layered composite laminate is investigated in detail. The material properties and the thickness are different in each layer. The analytical results obtained in this paper are exact and are expressed in a simple closed form, each mathematical term representing a physical transient wave. For the numerical calculation, a two-layered composite laminate is investigated. The transient shear stresses at different positions in the two layers are presented and compared with the corresponding static solution. The results of the numerical calculations are used to investigate the characteristic time during which the transient effect is important.

\section{STATEMENTS OF THE PROBLEM}

In the theory of elasticity there exists a special class of problems, known as plane problems (i.e. inplane and antiplane), which can be solved more readily than the general three-dimensional problem, since certain simplifying assumptions can be made in their treatment. There are many physical situations where such a reduction is indicated. In twodimensional plane problems the components of the stress tensor are independent of one of the coordinates, say $z$, and the displacement equations of motion can be split up into two uncoupled systems. If the deformation described only by a displacement distribution $w(x, y, t)$ is called an antiplane shear deformation, this is the case that we have discussed in this study. For this type of motion, shear waves apparently decouple completely from compressional waves.

Consider a stress-free composite laminate with $n$ layers, each layer differing in material properties and thickness, as depicted in Fig. 1. Each layer is assumed homogeneous and isotropic and perfect bounding is considered at the interfaces. Layer (1) denotes the top layer, on which the dynamic loading is applied, and layer $(n)$ is the bottom layer. A Cartesian coordinate system is defined in the body in such a way that the antiplane deformation is in the $z$-direction. The top face of the $n$-layered composite lies in the plane $y=0$ and $-\infty<x<\infty$. At time $t=0$, a dynamic concentrated antiplane force of magnitude $\sigma_{0}$ (per unit length in the $z$-direction) acts at $x=0, y=0$. The relevant stress components are denoted by $\sigma_{x z}$ and $\sigma_{y z}$, and the nonzero out-of-plane displacement is 


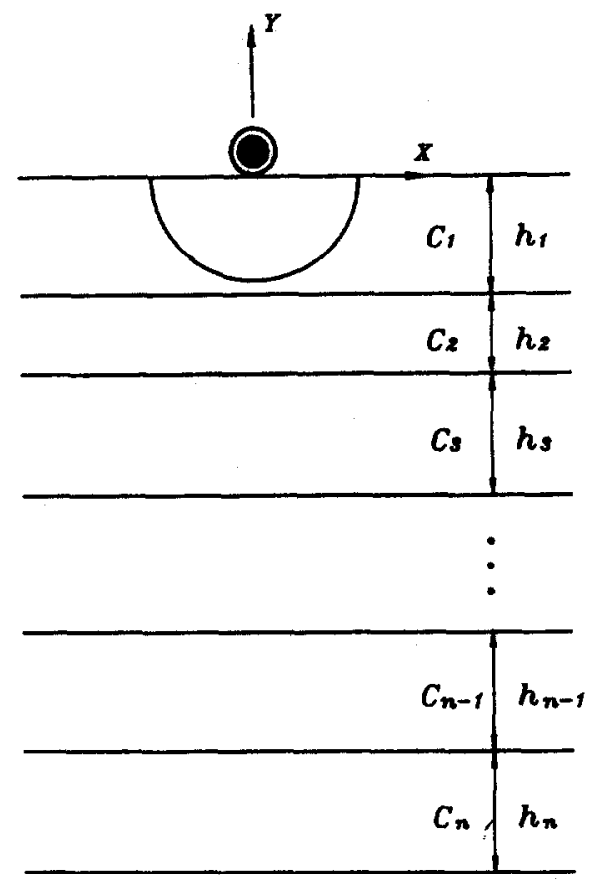

Fig. 1. Configuration and coordinate system of an $n$-layered medium.

denoted by $w$. Where $w(x, y, t)$ is the displacement in the $z$-direction, the corresponding stress components follow from Hooke's law as

$$
\sigma_{y z}=\mu \frac{\partial w}{\partial y}, \quad \sigma_{x z}=\mu \frac{\partial w}{\partial x}
$$

The boundary conditions on the top and bottom layers of the problem can be written as

$$
\begin{gathered}
\sigma_{y z}^{(1)}(x, 0, t)=\sigma_{0} \delta(x) H(t) \text { for }-\infty<x<\infty, \\
\sigma_{y z}^{(n)}\left(x,-h^{n}, t\right)=0 \text { for }-\infty<x<\infty
\end{gathered}
$$

where

$$
h^{n}=\sum_{j=1}^{n} h_{j}
$$

in which $h_{j}$ is the thickness of the $j$ th layer, $\delta(x)$ is the delta function and $H(t)$ is the Heaviside unit-step function. The solution for a more general applied dynamic loading can be obtained by a linear superposition. Perfect bonding along the interface is ensured by the stress and displacement continuity conditions of each layer:

$$
\begin{array}{ll}
\sigma_{y z}^{(i)}\left(x,-h^{i}, t\right)=\sigma_{y z}^{(i+1)}\left(x,-h^{i}, t\right) & 1 \leqslant i \leqslant n-1, \\
w^{(i)}\left(x,-h^{i}, t\right)=w^{(i+1)}\left(x,-h^{i}, t\right) & 1 \leqslant i \leqslant n-1 .
\end{array}
$$

The propagation of shear wave in a homogeneous, isotropic elastic medium is governed by the two-dimensional wave equation, 


$$
\frac{\partial^{2} w}{\partial x^{2}}+\frac{\partial^{2} w}{\partial y^{2}}=b^{2} \frac{\partial^{2} w}{\partial t^{2}}
$$

where $b$ is the slowness of the shear wave given by

$$
b=1 / c=\sqrt{p / \mu}
$$

in which $\mu$ and $\rho$ are the shear modulus and the mass density of the material, respectively, while $c$ is the shear wave velocity. This problem is solved by the application of integral transforms. Applying the Laplace transform of eqn (5) over time and the two-sided Laplace transform over $x$, the solutions of displacement and stresses in the Laplace transform domain are given in layer (i) by

$$
\begin{gathered}
\hat{w}^{(i)}=A_{i} \mathrm{e}^{p \alpha_{i} y}+B_{i}^{-p \alpha_{i} y}, \quad i=1, \ldots, n, \\
\hat{\sigma}_{y z}^{(i)}=p \mu_{i} \alpha_{i}\left(A_{i} \mathrm{e}^{p \alpha_{i} y}-B_{i} \mathrm{e}^{-p \alpha_{i} y}\right), \quad i=1, \ldots, n,
\end{gathered}
$$

where

$$
\alpha_{i}=\sqrt{b_{i}^{2}-\lambda^{2}}
$$

in which $p$ and $\lambda$ are the variables of the one-sided and the two-sided Laplace transform parameters, respectively. Constants $A_{i}$ and $B_{i}$ are to be determined through the transforms of the boundary and continuity conditions.

\section{TRANSIENT SOLUTIONS IN TRANSFORM DOMAIN FOR THE $n$-LAYERED MEDIUM}

This section is concerned with the derivation of the undetermined coefficients $A_{i}$ and $B_{i}$ in an $n$-layered medium. By using the general solutions obtained in the previous section and the continuity conditions in eqns (3) and (4), the relationship of the unknown constants $A_{i}$ and $B_{i}$ at each layer can be expressed as follows:

$$
T_{i}=\frac{B_{i}}{A_{i}} \mathrm{e}^{2 p \alpha_{i} h^{i}}=\frac{\gamma_{i j i+1}+T_{i+1} \mathrm{e}^{-2 p \alpha_{i+1} h_{i+1}}}{1+T_{i+1} \gamma_{i / i+1} \mathrm{e}^{-2 p \alpha_{i+1} h_{i+1}}},
$$

where

$$
\gamma_{i i+1}=\frac{\mu_{i} \alpha_{i}-\mu_{i+1} \alpha_{i+1}}{\mu_{i} \alpha_{i}+\mu_{i+1} \alpha_{i+1}}
$$

From the boundary condition of eqn (2), we have

$$
A_{n}=B_{n} \mathrm{e}^{2 p x_{n} h^{n}}
$$

Hence

$$
T_{n}=1
$$

The relationship of the unknown constants for different layers can be obtained from continuity conditions. The results are 


$$
\begin{gathered}
\frac{A_{i+1}}{A_{i}}=\frac{f_{i+1 / i} \mathrm{e}^{p\left(z_{i+1}-\alpha_{i}\right) h^{i}}}{1+\gamma_{i / i+1} T_{i+1} \mathrm{e}^{-2 p \alpha_{i+1} h_{i+1}}}=G_{i}, \\
\frac{B_{i+1}}{B_{i}}=\frac{T_{i+1} f_{i+1 / i} \mathrm{e}^{-2 p \alpha_{i+1} h_{i+1}}}{T_{i+1} \mathrm{e}^{-2 p x_{i+1} h_{i+1}}+\gamma_{i i+1}},
\end{gathered}
$$

wher:

$$
f_{i+1 / i}=\frac{2 \mu_{i} \alpha_{i}}{\mu_{i} \alpha_{i}+\mu_{i+1} \alpha_{i+1}}
$$

From the boundary condition at the top face as expressed in (1), we have

$$
A_{1}=B_{1}+\frac{\sigma_{0}}{p^{2} \mu_{1} x_{1}}
$$

Also from eqn (8), we have

$$
A_{1}=\frac{B_{1}}{T_{1}} \mathrm{e}^{2 p x, h_{1}}
$$

$A_{1}$ and $B_{1}$ can be obtained by solving eqns (13) and (14), which are

$$
\begin{aligned}
& A_{1}=\frac{1}{1-T_{1} \mathrm{e}^{-2 p z_{1} h_{1}}} \frac{\sigma_{0}}{p^{2} \mu_{1} \alpha_{1}}, \\
& B_{1}=\frac{T_{1} \mathrm{e}^{-2 p x_{1} h_{1}}}{1-T_{1} \mathrm{e}^{-2 p z_{1} h_{1}}} \frac{\sigma_{0}}{p^{2} \mu_{1} \alpha_{1}}
\end{aligned}
$$

Consequently all the unknown constants $A_{i}$ and $B_{i}$ for each layer can be obtained in terms of $A_{1}$ from the results expressed in eqns (8), (10), (11), (15) and (16). For example, the constants $A_{i}$ and $B_{i}$ at the $i$ th layer can be expressed as follow:

$$
\begin{aligned}
& A_{i}=\left\{\prod_{j=1}^{i-1} G_{j}\right\}_{1}=\left\{\prod_{j=1}^{i-1} G_{j}\right\} \frac{1}{1-T_{1} \mathrm{e}^{-2 p x_{1} h_{1}}} \frac{\sigma_{0}}{p^{2} \mu_{1} \alpha_{1}} \\
& B_{i}=T_{i} \mathrm{e}^{-2 p \alpha_{i} h^{i}} A_{i}=\left\{\prod_{j=1}^{i-1} G_{j}\right\} \frac{\mathrm{e}^{-2 p x_{i} h^{i}}}{1-T_{1} \mathrm{e}^{-2 p \alpha_{1} h_{1}}} \frac{\sigma_{0}}{p^{2} \mu_{1} \alpha_{1}}
\end{aligned}
$$

where

$$
\prod_{j=1}^{k} G_{j}=G_{1} G_{2} \ldots G_{k}
$$

Finally, from the result that $T_{n}=1$ and eqn (8), $T_{1}$ can be determined, and $G_{i}$ can also be deterimined from eqn (10). The solutions of displacement and shear stress in the Laplace transiform domain can then be obtained from eqns (6) and (7), respectively. 


\section{TRANSIENT SOLUTIONS FOR THE TWO-LAYERED MEDIUM}

In the previous section, the solutions in the Laplace transform domain are constructed for general $n$-layered medium. The transient full-field solutions can then be obtained by Laplace inversion of eqns (6) and (7). In order to explain the methodology for constructing the infinite reflected and refracted waves generated at each layer in time domain, a twolayered composite laminate is chosen to analyze the transient problem in detail. In this case we have $n=2$ and

$$
\begin{aligned}
A_{1} & =\frac{1}{1-T_{1} \mathrm{e}^{-2 p \alpha_{1} h_{1}}} \frac{\sigma_{0}}{p^{2} \mu_{1} \alpha_{1}} \\
& =\frac{\sigma_{0}}{p^{2} \mu_{1} \alpha_{1}} \sum_{m=0}^{\infty}\left\{T_{1} \mathrm{e}^{-2 p \alpha_{1} h_{1}}\right\}^{m},
\end{aligned}
$$

where

$$
\begin{gathered}
T_{1}=\frac{\gamma_{1 / 2}+\mathrm{e}^{-2 p \alpha_{2} h_{2}}}{1+\gamma_{1 / 2} \mathrm{e}^{-2 p \alpha_{2} h_{2}}} \\
=\gamma_{1 / 2}+f_{1 / 2} f_{2 / 1} \sum_{i=0}^{\infty} \gamma_{2 / 1}^{i} \mathrm{e}^{-2(i+1) p \alpha_{2} h_{2}}, \\
\gamma_{1 / 2}=1-f_{1 / 2}=-\gamma_{2 / 1}=\frac{\mu_{1} \alpha_{1}-\mu_{2} \alpha_{2}}{\mu_{1} \alpha_{1}+\mu_{2} \alpha_{2}}, \quad f_{2 / 1}=1-\gamma_{2 / 1}=\frac{2 \mu_{1} \alpha_{1}}{\mu_{1} \alpha_{1}+\mu_{2} \alpha_{2}} .
\end{gathered}
$$

Combining eqns (20) and (21), $A_{1}$ can be expressed in an infinite series, and the first few terms are

$$
\begin{aligned}
A_{1}= & \frac{\sigma_{0}}{p^{2} \mu_{1} \alpha_{1}} \sum_{m=0}^{\infty}\left\{\gamma_{1 / 2}+f_{1 / 2} f_{2 / 1} \sum_{i=1}^{\infty} \gamma_{2 / 1}^{i} \mathrm{e}^{-2(i+1) p \alpha_{2} h_{2}}\right\}^{m} \mathrm{e}^{-2 m p \alpha_{1} h_{1}} \\
= & \frac{\sigma_{0}}{p^{2} \mu_{1} \alpha_{1}}\left\{1+\gamma_{1 / 2} \mathrm{e}^{-2 p \alpha_{1} h_{1}}+f_{1 / 2} f_{2 / 1} \mathrm{e}^{-2 p\left(\alpha_{2} h_{2}+\alpha_{1} h_{1}\right)}\right. \\
& \left.\left.+\gamma_{2 / 1} f_{1 / 2} f_{2 / 1} \mathrm{e}^{-4 p \alpha_{2} h_{2}-2 p \alpha_{1} h_{1}}+\ldots\right\}\right\}
\end{aligned}
$$

Each term in eqn (22) represents propagating waves in the first layer and with the propagation direction along the negative $y$-axis. The first term in eqn (22) represents the incident wave generated by the dynamic concentrated loading at the top face. The second term represents the wave which is reflected from the interface and then reflected again from the upper free surface. The third term represents the wave which is refracted from the interface twice and then reflected from the upper free surface. The fourth term represents the wave which is refracted into the second layer, reflected from the interface and the lower free surface, refracted from the interface and then reflected from the upper free surface.

The value for $B_{1}$ is represented as follows:

$$
\begin{aligned}
B_{1}= & \frac{T_{1} \mathrm{e}^{-2 p \alpha_{1} h_{1}}}{1-T_{1} \mathrm{e}^{-2 p \alpha_{1} h_{1}}} \frac{\sigma_{0}}{p^{2} \mu_{1} \alpha_{1}}=\frac{\sigma_{0}}{p^{2} \mu_{1} \alpha_{1}} \sum_{m=0}^{\infty}\left\{T_{1} \mathrm{e}^{2 p \alpha_{1} h_{1}}\right\}^{m+1} \\
= & \frac{\sigma_{0}}{p^{2} \mu_{1} \alpha_{1}}\left\{\gamma_{1 / 2} \mathrm{e}^{-2 p \alpha_{1} h_{1}}+f_{1 / 2} f_{2 / 1} \mathrm{e}^{-2 p \alpha_{2} h_{2}-2 p \alpha_{1} h_{1}}\right. \\
& \left.+\gamma_{2 / 1} f_{1 / 2} f_{2 / 1} \mathrm{e}^{-4 p \alpha_{2} h_{2}-2 p \alpha_{1} h_{1}}+\ldots .\right\}
\end{aligned}
$$


Each term in eqn (23) represents propagating wave in the direction toward the positive $y$ axis for the first layer. The first term represents the reflected waves from the interface. The second term represents the wave which is refracted from the interface twice. The third term represents the wave which is refracted from the interface, reflected from the lower free surface and the interface of the second layer and refracted again from the interface.

The constants $A_{2}$ and $B_{2}$ at the second layer can also be obtained from the similar procedure as indicated for the first layer. The results are

$$
\begin{gathered}
A_{2}=\frac{\sigma_{0} f_{1 / 2} \mathrm{e}^{p\left(\alpha_{2}-\alpha_{1}\right) h_{1}}}{p^{2} \mu_{2} \alpha_{2}}\left\{\sum_{m=0}^{\infty}\left(\gamma_{1 / 2} T_{2} \mathrm{e}^{-2 p \alpha_{2} h_{2}}\right)^{m}\right\}\left\{\sum_{i=0}^{\infty}\left(T_{1} \mathrm{e}^{-2 p \alpha_{1} h_{1}}\right)^{i}\right\} \\
=\frac{\sigma_{0}}{p^{2} \mu_{2} \alpha_{2}}\left\{f_{1 / 2} \mathrm{e}^{p\left(\alpha_{2}-\alpha_{1}\right) h_{1}}+\gamma_{2 / 1} f_{1 / 2} \mathrm{e}^{p\left(\alpha_{2}-\alpha_{1}\right) h_{1}-2 p \alpha_{2} h_{2}}\right. \\
\left.\quad+\gamma_{2 / 1} \gamma_{2 / 1} f_{1 / 2} \mathrm{e}^{p\left(\alpha_{2}-\alpha_{1}\right) h_{1}-4 p \alpha_{2} h_{2}}+\ldots\right\}, \\
B_{2}=\frac{\sigma_{0}}{p^{2} \mu_{2} \alpha_{2}}\left\{\sum_{m=0}^{\infty}\left(T_{1} \mathrm{e}^{-2 p \alpha_{1} h_{1}}\right)^{m}\right\}\left\{f_{1 / 2} \mathrm{e}^{-p \alpha_{2}\left(h_{1}+2 h_{2}\right)-p \alpha_{1} h_{1}} \sum_{i=0}^{\infty}\left(T_{2} \gamma_{1 / 2} \mathrm{e}^{-2 p \alpha_{2} h_{2}}\right)^{m}\right\} \\
=\frac{\sigma_{0}}{p^{2} \mu_{2} \alpha_{2}}\left\{f_{1 / 2} \mathrm{e}^{-p \alpha_{2}\left(h_{1}+2 h_{2}\right)-p \alpha_{1} h_{1}}+\gamma_{2 / 1} f_{1 / 2} \mathrm{e}^{-p \alpha_{2}\left(h_{1}+4 h_{2}\right)-p \alpha_{1} h_{1}}\right. \\
+\gamma_{2 / 1} \gamma_{2 / 1} f_{1 / 2} \mathrm{e}^{-p \alpha_{2}\left(h_{1}+\sigma h_{2}\right)-p \alpha_{1} h_{1}}+\ldots . .
\end{gathered}
$$

We now focus our attention on the constructing of the transient solution for the shear stress $\sigma_{y z}^{(1)}$ of the first layer. The solution of $\sigma_{y z}^{(1)}$ in the transform domain is obtained from eqns (7), (22) and (23). The result is

$$
\begin{aligned}
\hat{\sigma}_{y z}^{(1)}= & p \mu_{1} \alpha_{1}\left(A_{1} \mathrm{e}^{p \alpha_{1} y}-B_{1} \mathrm{e}^{-p \alpha_{1} y}\right) \\
= & \sigma_{0} \frac{e^{p \alpha_{1} y}}{p}\left\{1+\gamma_{1 / 2} \mathrm{e}^{-2 p \alpha_{1} h_{1}}+f_{1 / 2} f_{2 / 1} \mathrm{e}^{-2 p\left(\alpha_{2} h_{2}+\alpha_{1} h_{1}\right)}+\ldots\right\} \\
& -\sigma_{0} \frac{\mathrm{e}^{-p \alpha_{1} y}}{p}\left\{\gamma_{1 / 2} \mathrm{e}^{-2 p \alpha_{1} h_{1}}+f_{1 / 2} f_{2 / 1} \mathrm{e}^{-2 p \alpha_{2} h_{2}-2 p \alpha_{1} h_{1}}+\ldots\right\} .
\end{aligned}
$$

The remaining task is to evaluate the inverse transforms of this expression. The inversion formula for the two-sided Laplace transform for the shear stress $\sigma_{y z}^{(1)}$ leads to

$$
\begin{aligned}
\tilde{\sigma}_{y z}^{(1)}=\frac{\sigma_{0}}{2 \pi i} \int_{\lambda_{1}-i \infty}^{\lambda_{1}+i \infty}\left\{\mathrm{e}^{p\left(\alpha_{1} y+\lambda x\right)}+\gamma_{1 / 2} \mathrm{e}^{p\left[\alpha_{1}\left(y-2 h_{1}\right)+\lambda x\right]}+\ldots\right\} \mathrm{d} \lambda \\
-\frac{\sigma_{0}}{2 \pi i} \int_{\lambda_{1}-i \infty}^{\lambda_{1}+i \infty}\left\{\gamma_{1 / 2} \mathrm{e}^{-p\left[\alpha_{1}\left(y+2 h_{1}\right)-\lambda x\right)}+f_{1 / 2} f_{2 / 1} \mathrm{e}^{-p\left[\alpha_{1}\left(y+2 h_{1}\right)+2 \alpha_{2} h_{2}-\lambda x\right]}\right. \\
\\
\left.\quad+\gamma_{1 / 2} \gamma_{1 / 2} \mathrm{e}^{-p\left[\alpha_{1}\left(y+4 h_{1}\right)-\lambda x\right]}+\ldots\right\} \mathrm{d} \lambda .
\end{aligned}
$$

The overbar symbol is used to denote the transform on time $t$. The exact transient solution in time domain can then be found by inverting the Laplace transform by Cagniardde Hoop method. The idea of the Cagniard-de Hoop method is to deform the path of integration in the $\lambda$-plane in such a manner that the inverse Laplace transform of the integral along the new path of integration can be obtained by inspection. The desired path of integration in the $\lambda$-plane for the first few waves is defined by the following equations: 


$$
\begin{gathered}
\text { C.-C. Ma and K.-C. Huang } \\
-\left(b_{1}^{2}-\lambda^{2}\right)^{1 / 2} y-\lambda x=t, \\
\left(b_{1}^{2}-\lambda^{2}\right)^{1 / 2}\left(y+2 h_{1}\right)-\lambda x=t, \\
\left(b_{1}^{2}-\lambda^{2}\right)^{1 / 2}\left(y+2 h_{1}\right)+2\left(b_{2}^{2}-\lambda^{2}\right)^{1 / 2} h_{2}-\lambda x=t, \\
-\left(b_{1}^{2}-\lambda^{2}\right)^{1 / 2}\left(y-2 h_{1}\right)-\lambda x=t, \\
\left(b_{1}^{2}-\lambda^{2}\right)^{1 / 2}\left(y+4 h_{1}\right)-\lambda x=t .
\end{gathered}
$$

Equations (28), (29), (31) and (32) can be solved analytically to describe a hyperbola. We cannot solve eqn (30) analytically for $\lambda$, but from the formulation of a quartic algebraic equation, the roots can be obtained explicitly. The roots of equations (28)-(32) are denoted as $\lambda_{1}, \lambda_{2}, \lambda_{3}, \lambda_{4}$ and $\lambda_{5}$, respectively. The arrival time of each waves in eqns (28)-(32) is denoted as $t_{1}, t_{2}, t_{3}, t_{4}$, and $t_{5}$, which can be determined from the condition when the imaginary part of $\lambda_{1}, \lambda_{2}, \lambda_{3}, \lambda_{4}$ and $\lambda_{5}$ vanishes, respectively. Equations (28)-(32) represent the $i, r, f r f, r r$ and $r r$ wave, respectively. series,

The transient solution of $\sigma_{y z}^{(1)}$ in the upper layer can be written as a form of an infinite

$$
\sigma_{y z}^{(1)}(x, y, t)=\frac{\sigma_{0}}{\pi} \sum_{i=1}^{\infty} \operatorname{Im}\left\{F_{i}\left(\lambda_{i}\right) \frac{\partial \lambda_{i}}{\partial t} H\left(t-t_{i}\right)\right\}
$$

The first few terms are

$$
\begin{aligned}
& F_{1}\left(\lambda_{1}\right)=1, \\
& F_{2}\left(\lambda_{2}\right)=-\gamma_{1 / 2}\left(\lambda_{2}\right), \\
& F_{3}\left(\lambda_{3}\right)=1-\gamma_{1 / 2}^{2}\left(\lambda_{3}\right), \\
& F_{4}\left(\lambda_{4}\right)=\gamma_{1 / 2}\left(\lambda_{4}\right), \\
& F_{5}\left(\lambda_{5}\right)=-\gamma_{1 / 2}^{2}\left(\lambda_{5}\right),
\end{aligned}
$$

where

$$
\gamma_{1 / 2}(\lambda)=\frac{\mu_{1} \sqrt{b_{1}^{2}-\lambda^{2}}-\mu_{2} \sqrt{b_{2}^{2}-\lambda^{2}}}{\mu_{1} \sqrt{b_{1}^{2}-\lambda^{2}}+\mu_{2} \sqrt{b_{2}^{2}-\lambda^{2}}} .
$$

From the similar procedure as indicated above to construct the transient solution in the first layer, the transient solution for $\sigma_{y z}^{(2)}$ in the second layer is found to be

$$
\sigma_{y z}^{(2)}(x, y, t)=\frac{\sigma_{0}}{\pi} \sum_{i=1}^{\infty} \operatorname{Im}\left\{F_{i}^{*}\left(\lambda_{i}^{*}\right) \frac{\partial \lambda_{i}^{*}}{\partial t} H\left(t-t_{i}^{*}\right)\right\}
$$

The first few terms are

$$
\begin{aligned}
& F_{1}^{*}(\lambda)=f_{1 / 2}(\lambda), \\
& F_{2}^{*}(\lambda)=\gamma_{1 / 2}(\lambda) f_{1 / 2}(\lambda), \\
& F_{3}^{*}(\lambda)=f_{1 / 2}(\lambda),
\end{aligned}
$$




$$
F_{4}^{*}(\lambda)=f_{1 / 2}(\lambda) \gamma_{2 / 1}(\lambda),
$$

where

$$
f_{1 / 2}(\lambda)=\frac{2 \mu_{2} \sqrt{b_{2}^{2}-\lambda^{2}}}{\mu_{1} \sqrt{b_{1}^{2}-\lambda^{2}}+\mu_{2} \sqrt{b_{2}^{2}-\lambda^{2}}}
$$

and $\lambda_{1}^{*}, \lambda_{2}^{*}, \lambda_{3}^{*}$ and $\lambda_{4}^{*}$ are the roots for the following respective equations:

$$
\begin{gathered}
-\left(b_{2}^{2}-\lambda^{2}\right)^{1 / 2}\left(y+h_{1}\right)+\left(b_{1}^{2}-\lambda^{2}\right)^{1 / 2} h_{1}-\lambda x=t, \\
-\left(b_{2}^{2}-\lambda^{2}\right)^{1 / 2}\left(y+h_{1}\right)-3\left(b_{1}^{2}-\lambda^{2}\right)^{1 / 2} h_{1}-\lambda x=t, \\
\left(b_{2}^{2}-\lambda^{2}\right)^{1 / 2}\left(y+h_{1}+2 h_{2}\right)+\left(b_{1}^{2}-\lambda^{2}\right)^{1 / 2} h_{1}-\lambda x=t, \\
-\left(b_{2}^{2}-\lambda^{2}\right)^{1 / 2}\left(y+h_{1}-2 h_{2}\right)+\left(b_{1}^{2}-\lambda^{2}\right)^{1 / 2} h_{1}-\lambda x=t .
\end{gathered}
$$

The arrival times $t_{1}^{*}, t_{2}^{*}, t_{3}^{*}$ and $t_{4}^{*}$ are determined from the condition when the imaginary part of $\lambda_{1}^{*}, \lambda_{2}^{*}, \lambda_{3}^{*}$ and $\lambda_{4}^{*}$ vanishes, respectively. Equations (35)-(38) represent the $f, r r f, f r$ and $f r r$ wave, respectively.

In order to understand the detail for the transient response approaches to the correspondent static value, the static solutions of the two-layered medium for applying a static point loading $\sigma_{0}$ at the top surface are obtained by using the Fourier transform method. The result of the displacement can be expressed as the following form:

$$
w^{(1)}=\frac{\sigma_{0}}{\pi \mu_{1}} \sum_{n=0}^{\infty}\left\{\hat{\gamma}_{1 / 2}+\hat{f}_{1 / 2} \hat{f}_{2 / 1} \sum_{m=0}^{\infty} \hat{\gamma}_{2 / 1}^{m} G(0, m+1)\right\}^{n} G(n, 0)
$$

where

$$
G(i, j)=\ln \left\{\left[x^{2}+\left(y-2 j h_{1}-2 i h_{2}\right)^{2}\right]\left[x^{2}+\left(y+2 j h_{1}+2 i h_{2}\right)^{2}\right]\right\}, \quad G(0,0)=\ln \left(x^{2}+y^{2}\right) .
$$

The product of the function $G$ should be evaluated by the following rule:

$$
G(a, b) G(c, d)=G(a+c, b+d)
$$

and

$$
w^{(2)}=\frac{\sigma_{0} \hat{f}_{1 / 2}}{\pi \mu_{2}}\left\{\sum_{l=0}^{\infty} \hat{\gamma}_{2 / 1}^{l} P(0, l)\right\}\left\{\sum_{n=0}^{\infty}\left[\hat{\gamma}_{1 / 2}+\hat{f}_{1 / 2} \hat{f}_{2 / 1} \sum_{m=0}^{\infty} \hat{\gamma}_{2 / 1}^{m} P(0, m+1)\right]^{n} P(n, 0)\right\},
$$

where

$$
\begin{array}{r}
P(i, j)=\ln \left\{\left[x^{2}+\left(y-2 j h_{1}-2 i h_{2}\right)^{2}\right]\left[x^{2}+\left(y+2(j+1) h_{1}+2(i+1) h_{2}\right)^{2}\right]\right\}, \\
P(a, b) P(c, d)=P(a+c, b+d),
\end{array}
$$

in which 


$$
\begin{gathered}
\hat{\gamma}_{1 / 2}=-\hat{\gamma}_{2 / 1}=\frac{\mu_{1}-\mu_{2}}{\mu_{1}+\mu_{2}}, \\
\hat{f}_{1 / 2}=\frac{2 \mu_{2}}{\mu_{1}+\mu_{2}}, \quad \hat{f}_{2 / 1}=\frac{2 \mu_{1}}{\mu_{1}+\mu_{2}} .
\end{gathered}
$$

The solution for the shear stress $\sigma_{y z}$ is

$$
\sigma_{y z}^{(1)}=\frac{\sigma_{0}}{\pi} \sum_{n=0}^{\infty}\left\{\hat{\gamma}_{1 / 2}+\hat{f}_{1 / 2} \hat{f}_{2 / 1} \sum_{n=0}^{\infty} \hat{\gamma}_{2 / 1}^{m} g(0, m+1)\right\}^{n} g(n, 0),
$$

where

$$
\begin{gathered}
g(i, j)=\frac{y-2 j h_{1}-2 i h_{2}}{x^{2}+\left(y-2 j h_{1}-2 i h_{2}\right)^{2}}+\frac{y+2 j h_{1}+2 i h_{2}}{x^{2}+\left(y+2 j h_{1}+2 i h_{2}\right)^{2}}, \\
g(a, b) g(c, d)=g(a+c, b+d), \quad g(0,0)=\frac{y}{x^{2}+y^{2}}, \\
\sigma_{y z}^{(2)}=\frac{\sigma_{0} \hat{f}_{1 / 2}}{\pi}\left\{\sum_{i=0}^{\infty} \hat{\gamma}_{2 / 1}^{l} p(0, l)\right\}\left\{\sum_{n=0}^{\infty}\left[\hat{\gamma}_{1 / 2}+\hat{f}_{1 / 2} \hat{f}_{2 / 1} \sum_{m=0}^{\infty} \hat{\gamma}_{2 / 1}^{m} p(0, m+1)\right]^{n} p(n, 0)\right\},
\end{gathered}
$$

where

$$
\begin{aligned}
& p(i, j)=\frac{y-2 j h_{1}-2 i h_{2}}{x^{2}+\left(y-2 j h_{1}-2 i h_{2}\right)^{2}}+\frac{y+2(j+1) h_{1}+2(i+1) h_{2}}{x^{2}+\left[y+2(j+1) h_{1}+2(i+1) h_{2}\right]^{2}}, \\
& \quad p(a, b) p(c, d)=p(a+c, b+d) .
\end{aligned}
$$

\section{NUMERICAL RESULTS}

With the explicit transient solution obtained in the previous section, the transient response will be investigated and discussed in detail in this section. For the numerical investigation, a two-layered composite laminate is considered. The ratio of the thickness for the two-layered medium is chosen to be $h_{2} / h_{1}=0.8$. At time $t=0$, an antiplane dynamic concentrated loading with magnitude $\sigma_{0}$ is applied at the top surface of the first layer. The time dependence of the loading is represented by the Heaviside step function $H(t)$. The wave fronts in this two-layered medium at the early time are shown in Figs 2 and 3. In Fig.

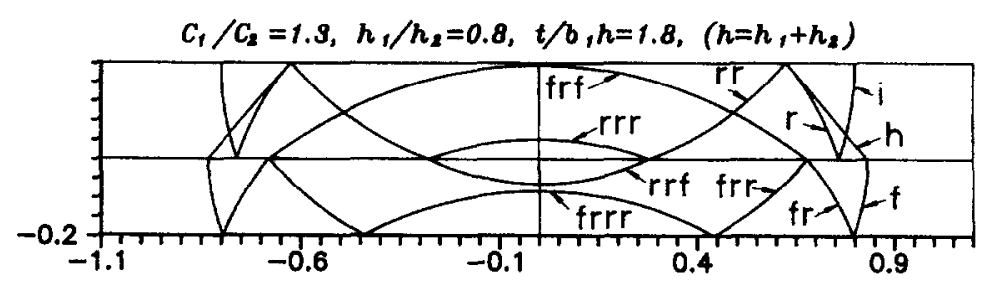

Fig. 2. Wave fronts of the incident, reflected and refracted waves of a two-layered medium for $c_{2} / c_{1}=1.3$

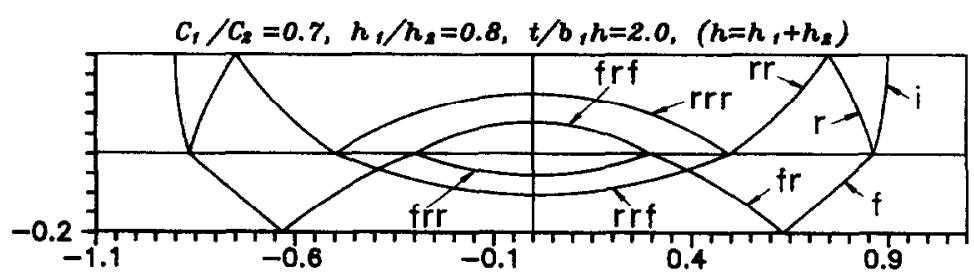

Fig. 3. Wave fronts of the incident, reflected and refracted waves of a two-layered medium for $c_{2} / c_{1}=0.7$. 


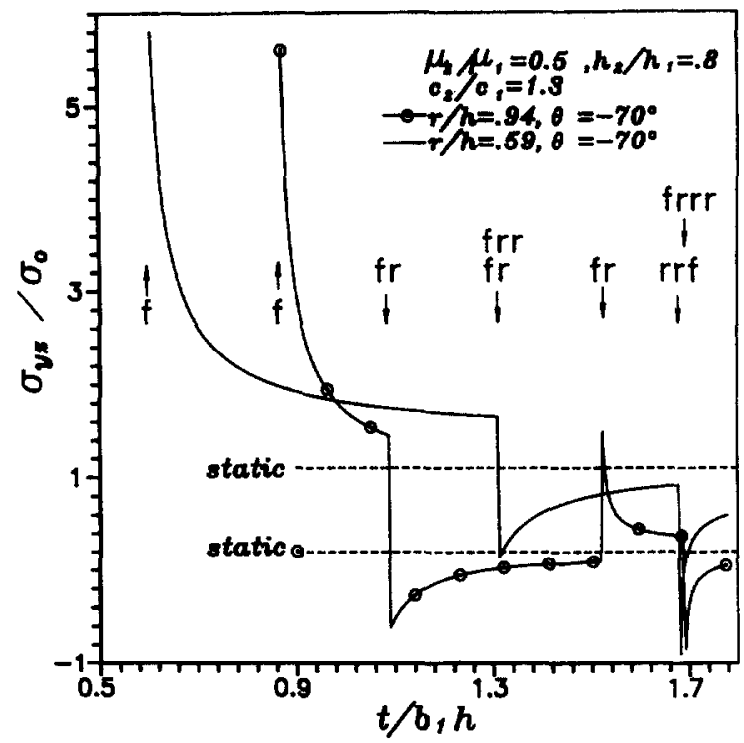

Fig. 4. Transient shear stress history at different field points in the second layer for $c_{2} / c_{1}=1.3$.

2 , the ratio of the shear wave speed $c_{2} / c_{1}$ equals 1.3 and a head wave will be generated in the first layer. Only the pure reflected waves (i.e. $i$ wave, $r$ wave, $r r$ wave and $r r$ wave) in the first layer are cylindrical curves, the head wave is an inclined straight line, and all other wave fronts are smooth curves which are constructed by numerical calculations. The transient shear stresses at different positions in the two-layered medium are presented in Figs 4-8. Figures 4-6 present the transient shear stresses for the case that the shear wave velocity in the first layer is smaller than that in the second layer. Figure 4 represents the transient stress history of two points, one is at the interface $(r / h=0.59)$ and the other one is located in the second layer. The time has been normalized by dividing by $b_{1} h\left(h=h_{1}+h_{2}\right)$ and the arrival time for each wave front is indicated in the figure. The static solutions obtained from eqns (41) and (42) are also presented in the same figure and are used to compare with the transient response. In Fig. 5, the transient responses for the point in the first layer $(r / h=0.33)$ and the point at the interface $(r / h=0.56)$, which is directly below the applied loading, are presented. In order to see the contribution for the head wave, a particular point in the first layer is chosen such that the head wave will pass this point, and the transient result for this point is shown in Fig. 6. Figures 7-8 show the results for the

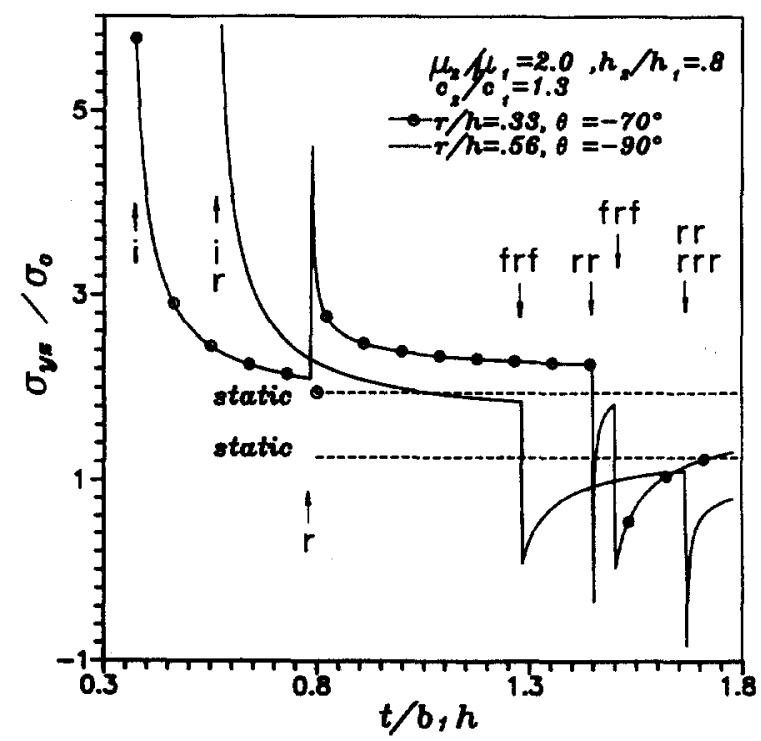

Fig. 5. Transient shear stress history at different field points in the first layer for $c_{2} / c_{1}=1.3$. 


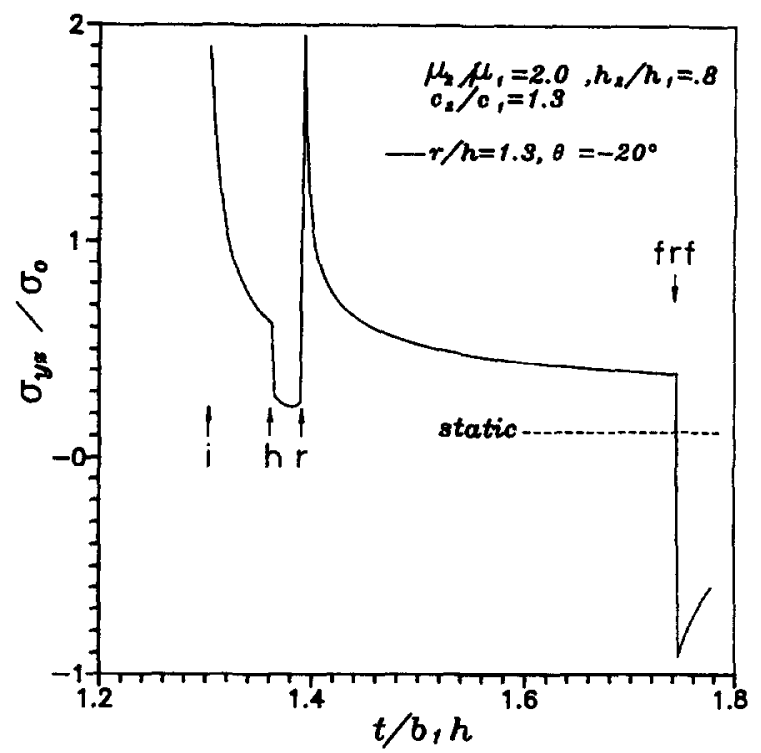

Fig. 6. Transient shear stress history at different field points in the first layer for $c_{2} / c_{1}=1.3$.

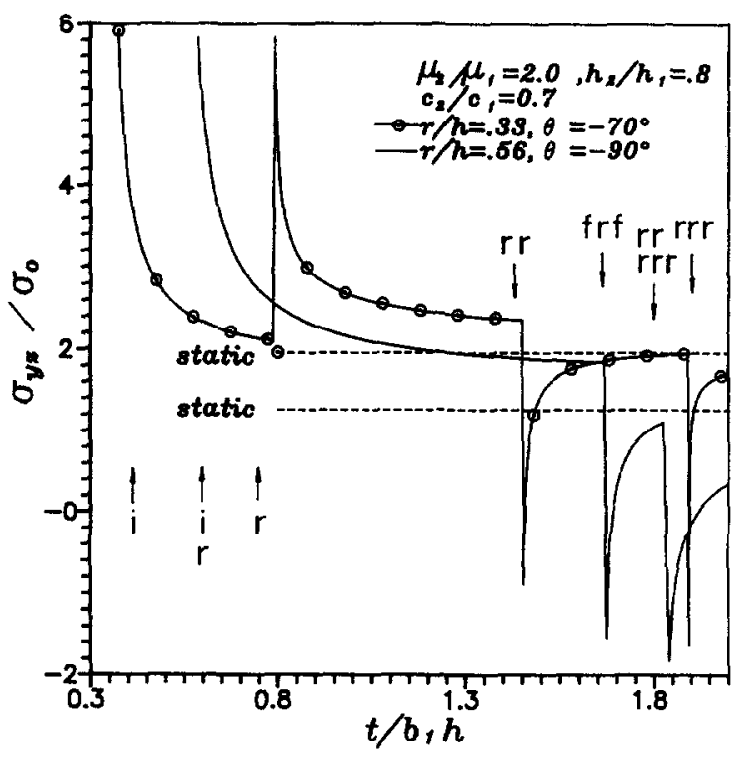

Fig. 7. Transient shear stress history at different field points in the first layer for $c_{2} / c_{1}=0.7$.

case that the shear wave velocity in the first layer is larger than that in the second layer. The static solutions are also shown in these figures and the transient solution approaches the correspondent static value as time increases. It is worthy to note that for applying point force of Heaviside function dependence, the stress fields behave as the square root singularity at all the reflected and refracted wave fronts, except for the case of the head wave.

\section{CONCLUSIONS}

Most of the studies for wave propagation in a layered medium focus on the determination of the dispersion relation or the frequency equation due to a harmonic oscillation. Because of the difficulty in analyzing the transient response of an infinite number of reflected and refracted waves in a finite-layered medium, the only available solutions are obtained for the transient plane wave propagating in a periodically layered medium. In this study, a general methodology is proposed to construct the transient solutions of a layered composite laminate subjected to dynamic impact loading, which will generate infinite number of 


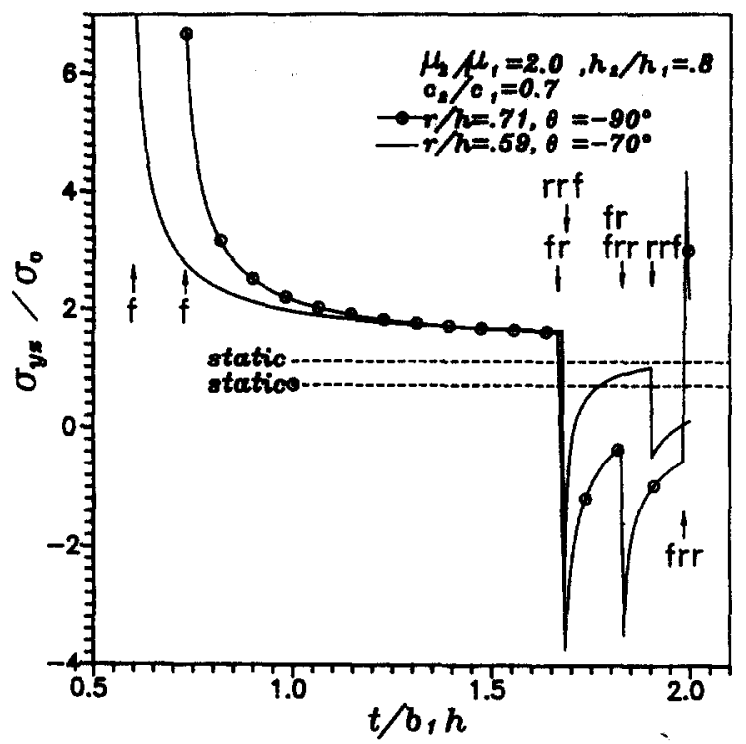

Fig. 8. Transient shear stress history at different field points in the second layer for $c_{2} / c_{1}=0.7$.

nonplanar reflected and refracted waves in each layer. The Cagniard-de Hoop inverse method is used, enabling us to investigate in detail the structure of the wave pattern and transient solutions in time domain. The analytical results obtained in this study are exact and are expressed in a simple closed series form, each mathematical term representing a physical transient wave. The methodology provided in this study has already been successfully extended to solve more complex types of problem, which deal with the transient wave propagation in layered medium, by applying inplane dynamic loading by $\mathrm{Ma}$ and Huang (1994).

In order to investigate the characteristic time after which the transient effect can be neglected, numerical results of shear stress based on the transient analysis are presented and compared to the corresponding static solution. The transient analysis in this study tends toward the corresponding static value as expected. Generally speaking, the dynamic transient effect is important under dynamic loading conditions. The dynamic response of stress in the transient period is much larger than the static value. In the transient period, the stress will change radically when the reflected or the refracted wave arrives. The transient value of stress will tend toward the static value after first few waves have passed.

Acknowledgement-The financial support of the authors from the National Science Council, Republic of China, through Grant NSC 81-0422-E-002-557 to National Taiwan University is gratefully acknowledged.

\section{REFERENCES}

Cagniard, L. (1962). Reflection and refraction of progressive waves. McGraw-Hill, New York.

Drumheller, D. S. and Bedford, A. (1973). On a continuum theory for a laminated medium. ASME J. Appl. Mech. 40, pp. 527-532.

Garvin, W. W. (1956). Exact transient solution of the buried line source problem. Proc. R. Soc. Lond. A234, 528541.

Hegemier, G. A. and Nayfeh, A. H. (1973). A continuum theory for wave propagation in laminated composites. Case 1: propagation normal to the laminates. ASME J. Appl. Mech. 40, 503-510.

Hegemier, G. A. and Bache, T. C. (1973). A continuum theory for wave propagation in laminated composites. Case 2: propagation parallel to laminates. J. Elast. 3, 125-140.

de Hoop, A. T. (1961). A modification of Cagniard's method for solving the seismic pulse problem. Appl. Sci. Res. 8, 349-356.

Lamb, H. (1904). On the propagation of tremors over the surface of an elastic solid. Phil. Trans. R. Soc. Lond. A203, 1-42.

Lapwood, E. R. (1949). The disturbance due to a line source in a semi-infinite elastic medium. Phil. Trans. $R$. Soc. Lond. A242, 63-100.

Ma, C. C. and Huang, K. C. (1994). Analytical transient analysis of layered composite medium subjected to dynamic impact loadings. Submitted to Int. J. Solids Structures.

Mukunoki, I. and Ting, T. C. T. (1980). Transient wave propagation normal to the layering of a finite layered medium. Int. J. Solids Structures 16, 239-251. 
Nakano, H. (1925). On Rayleigh waves. Jap. J. Astr. Geophys. 2, 233-326.

Nemat-Nasser, S. and Yamada, M. (1981). Harmonic waves in layered transversely isotropic composites. J. Sound Vibr. 79, 161-170.

Stern, M., Bedford, A. and Yew, C. H. (1971). Wave propagation in viscoelastic laminates. ASME J. Appl. Mech. $38,448-454$.

Sun, C. T., Achenbach, J. D. and Herrmann, G. (1968). Time-harmonic waves in a stratified medium propagating in the direction of the layering. ASME J. Appl. Mech. 35, 408-411.

Tang, Z. and Ting, T. C. T. (1985). Transient waves in a layered anisotropic elastic medium. Proc. R. Soc. Lond. A397, 67-85.

Ting, T. C. T. and Mukunoki, I. (1979). A theory of viscoelastic analogy for wave propagation normal to the layering of a layered medium. ASME J. Appl. Mech. 46, 329-336.

Tsai, C. H. and Ma, C. C. (1991). Exact transient solutions of buried dynamic point forces and displacement jumps for an elastic half space. Int. J. Solids Structures 28, 955-974. 